\title{
Dynamic formation of SEBS copolymer submicrometric structures
}

\author{
Michele A. Salvador ${ }^{\text {a }}$, Andrea G.C. Bianchi ${ }^{\text {a, } *}$, Marcelo A. Pereira-da-Silva ${ }^{\text {b,c }}$, Antonio J.F. Carvalho ${ }^{\text {d, }}$ \\ Roberto M. Faria ${ }^{\mathrm{b}}$ \\ ${ }^{a}$ Physics Department, University Federal of Ouro Preto, Campus Morro do Cruzeiro, Ouro Preto - 35400-000, MG, Brazil \\ ${ }^{\mathrm{b}}$ Institute of Physics of São Carlos, University of São Paulo, Av. Trabalhador São-carlense, 400, São Carlos -1356-970, SP, Brazil \\ ${ }^{\mathrm{c}}$ UNICEP - Centro Universitário Central Paulista, R. Miguel Petroni, 5111, São Carlos -13563-470- SP, Brazil \\ ${ }^{\mathrm{d}}$ Department of Material Engineering, University of São Paulo, Av. Trabalhador São-carlense, 400, São Carlos 1356-970, SP, Brazil
}

\section{A R T I C L E I N F O}

\section{Article history:}

Received 7 April 2010

Received in revised form

21 June 2010

Accepted 30 June 2010

Available online 7 July 2010

\section{Keywords:}

Block copolymer

Thin films

Phase separation

\begin{abstract}
A B S T R A C T
Highly ordered A-B-A block copolymer arrangements in the submicrometric scale, resulting from dewetting and solvent evaporation of thin films, have inspired a variety of new applications in the nanometric world. Despite the progress observed in the control of such structures, the intricate scientific phenomena related to regular patterns formation are still not completely elucidated. SEBS is a standard example of a triblock copolymer that forms spontaneously impressive pattern arrangements. From macroscopic thin liquid films of SEBS solution, several physical effects and phenomena act synergistically to achieve well-arranged patterns of stripes and/or droplets. That is, concomitant with dewetting, solvent evaporation, and Marangoni effect, Rayleigh instability and phase separation also play important role in the pattern formation. These two last effects are difficult to be followed experimentally in the nanoscale, which render difficulties to the comprehension of the whole phenomenon. In this paper, we use computational methods for image analysis, which provide quantitative morphometric data of the patterns, specifically comprising stripes fragmentation into droplets. With the help of these computational techniques, we developed an explanation for the final part of the pattern formation, i.e. structural dynamics related to the stripes fragmentation.
\end{abstract}

(ㄷ) 2010 Elsevier Ltd. All rights reserved.

\section{Introduction}

The patterned structures formed from dewetting thin films of block copolymer (BC) in solution are developing a promising branch on template technology at nanoscale, but the underlying science is still opened to be explored [1-3]. The application of such structures have been considered as a potential alternative for the soft lithography applied in nano and microelectronics as well as in other branches of contemporaneous technologies such as microfluidics and biotechnology [4,5]. It is widely known that symmetrical ABA triblock copolymers (TBC), depending on the A/B molar (volume fraction) ratio, derive different thermodynamically stable microphases: from lamellae to gyroidal morphologies [6-8]. More recently, the dewetting of thin films of poly(styrene)-b-poly(ethene-co-butene-1)-b-poly(styrene) (SEBS) solutions, under the influence of solvent evaporation, resulted in self-organized patterns formed by submicrometric droplets and/

\footnotetext{
* Corresponding author. Tel.: +55 3559 1742; fax: +55 35591667.

E-mail address: andrea@iceb.ufop.br (A.G.C. Bianchi).
}

or stripes arrangements. These structures were found for a relatively low concentration of SEBS in the solution; above a certain concentration value the final result was a uniform film covering the whole substrate [9]. The explanation for the stripes formation comes from a non-classical dewetting process in which each point at the three-phase contact line recedes towards the center of the liquid-drop, while a gradient of copolymer concentration generates an undulation at the vicinity of the contact line (Marangoni effect) [10]. Thus, long and parallel fingers are formed as the film shrinks. This phenomenon, called "regular fingering formation", can, in many cases, generate a well-arranged pattern of stripes. However, following the formation of fingers, they can be fragmented into droplets due to a phenomenon called Rayleigh instability. It was also observed that, depending on the receding speed of the contact line, the formation of droplets can occur directly from the contact line rupture, preventing the fingers formation [10].

A similar sequence of events had also been observed for homopolymer solutions [5], but for block copolymers impressive self-organized arrangements have been reported, as the regular patterns composed of stripes or droplets $[9,11]$. Such 
self-organized patterns were certainly caused by the dewetting phenomenon with a contribution of the solvent evaporation, but should be strongly affected by antagonistic interactions between block-segments of the copolymer, interactions that increase progressively as the solvent evaporates. On the other hand, studies carried out with thin films of polystyrene-butadiene-polystyrene copolymer - a copolymer with very similar properties to those of the SEBS - deposited on solid substrates found regular cylindrical polystyrene structures oriented either parallel or perpendicularly to the substrate [12]. Depending on the thickness of the film and the evaporation rate of the solvent, the thermodynamic equilibrium, that involves both internal interactions and the interaction between the film and the substrate, defines the orientation of the PS-cylinders [13]. In very thin films, PS-cylinders become perpendicularly arranged to the surface of the substrate, but as the number of degrees of freedom rises, what happens as the thickness increases, the system progresses to a parallel orientation. Despite the progress in the understanding of self-organized patterns of SEBS in submicrometric scales, the entire mechanism involved in such phenomenon, however, remains to be fully elucidated. The most obscure point is the finger fragmentation phenomenon that occurs just after the formation of the fingers, effects that happens when the amount of solvent is very small. This paper intends to give a contribution to the study of SEBS patterns, focusing on the geometrical and morphological information of fingers (called stripes from now on) and droplets. For this, we make use of the potential of quantitative computer techniques.

Due to the versatility and accuracy of the current image processing techniques, they have emerged as promising tools for microscopy image analysis and have been used to enrich investigations of patterned nanostructures [14], specially for morphological and surface analysis of thin films [15-18]. For this reason, this technique is adequate for obtaining important quantitative data to help in the investigation of the dynamic evolution of submicrometric SEBS structures, and can replace with great advantage the traditional methods provided by the commercial microscope software. In addition, it can process a large volume of imaging data in a semi-automated way, which allows for the study of statistical behavior of the collected data.

\section{Materials and methods}

\subsection{Materials}

We use a commercial SEBS (Kraton ${ }^{\circledR} \mathrm{G}$ 1560) with weight ratio of $15(\mathrm{~S}): 70(\mathrm{~EB}): 15(\mathrm{~S})$. The following values were obtained by gel permeation chromatography (GPC) in THF solution with polystyrene standards: $M_{\mathrm{w}}=112.000 \mathrm{~g} \mathrm{~mol}^{-1}$ and $M_{\mathrm{w}} / M_{\mathrm{n}}=1.15$. Toluene was used as solvent, and mica substrate as cleaved was used as solid substrate. The advantage of mica comes from its very low roughness and because its surface is relatively chemically inert [19].

\subsection{Sample preparation}

A layer of mica freshly cleaved twice was then plunged for few seconds in a SEBS/toluene solution $\left(\sim 0.10 \mathrm{~g} \mathrm{~L}^{-1}\right)$; enough time to form a thin liquid film that covers the whole area of the substrate. This dip coating procedure was carried out at environmental conditions with temperatures between $20{ }^{\circ} \mathrm{C}$ and $25{ }^{\circ} \mathrm{C}$ and relative humidity between $30 \%$ and $40 \%$. The liquid film is then left in the laboratory environment for several minutes for evaporating the toluene. Toluene is an appropriate
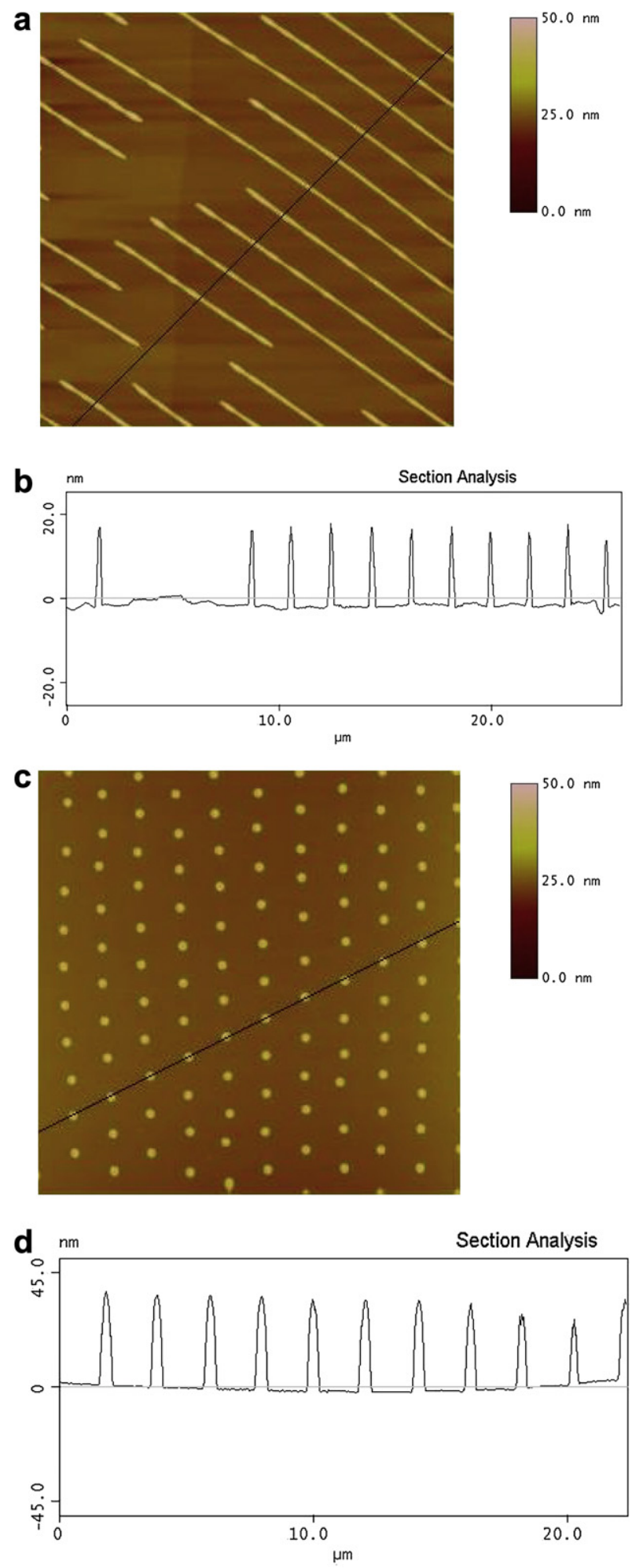

Fig. 1. AFM height images of self-assembled copolymer, illustrating the typical patterns: stripes (a) and droplets (c). The scan range was $20 \mu \mathrm{m} \times 20 \mu \mathrm{m} \times 50 \mathrm{~nm}$. Figure (b) shows the profile of (a), with maximum peak nearly $20 \mathrm{~nm}$, and (d) a profile analysis of image (c), with maximum peak ca. $43 \mathrm{~nm}$. The patterns were produced by dip coating from a $0.1 \mathrm{~g} / \mathrm{L}$ SEBS toluene solution.

solvent for both PS and PEB, mainly because it does not influence the initial molecular interactions, that is, when the solution is still diluted. The dynamics of molecular actions and the final structural arrangement depend on the specific interactions 
between each of the components of the block copolymer and the substrate.

\section{3. $A F M$}

AFM height images of the dried sample were obtained by tapping-mode atomic force microscopy (TM-AFM) (Nanoscope III, Digital Instruments). AFM analysis was carried out in a MultiMode Nanoscope 3 from Digital Instruments using intermittent contact mode (tapping-mode) with a silicon tip at $330.000 \mathrm{~Hz}$ oscillating frequency and scan speed $10 \mathrm{~mm} / \mathrm{s}$. The images were captured in the retrace scan direction for both height and phase images.

\subsection{Image processing}

AFM images were analyzed by calculating a set of semiautomated morphometric measurements such as height, area, width and distance of stripes and droplets of the SEBS structures. The initial processing step is the segmentation of objects (stripes or droplets) to be investigated. By applying an adaptive threshold algorithm to the AFM images, different morphological objects have been separated from the background, generating black (background) and white (objects) images [20]. The objects recognition is then extracted using the connected components labeling. This labeling technique groups pixels (the smallest cell of the image) into elements that share pixels of similar intensities [20]. The pattern recognition for objects and the labeling of various disjoint and connected components are decisive to a reliable automated image analysis, for example, the area calculation results in the sum of pixels for each object as well as mean height measures. The distance between neighboring objects, on the other hand, is measured according to the AFM image patterns, i.e. for droplets it is calculated as the distance between the centers of two objects, while for stripes it is obtained by a semi-automated procedure. In this procedure a line orthogonal to the stripes is determined over a segmented image, and the distance is obtained by the extension of the black pixels. For this paper in particular, these techniques contribute greatly for the specific dynamics related to the fragmentation of stripes in droplets.

\section{Results}

\subsection{AFM images}

Fig. 1 shows AFM images of self-organized structures found in different regions of the same sample. Fig. 1a exhibits a region patterned with parallel stripes, while in another region a quasiperfect 2D hexagonal lattice constituted by submicrometric droplets was recorded (Fig. 1b) [9]. Fig. 1c and d show, using a section profile analysis (a height profile), how regular these structures are, as well as the uniformity of shapes of stripes and droplets. Those images are the final result after dewetting and solvent evaporation of a thin liquid film that was formed by dipping the mica substrate in a SEBS/toluene solution for few seconds [9]. SEBS stripes and droplets adhered to the mica surface after the complete evaporation of the solvent, and inside each stripe or droplet the phase separation is such that parallel cylindrical structures of polystyrene remain embedded in the poly(ethene-co-butene) matrix, as shown in the AFM phase image (Fig. 2). It is clear that some perpendicular cylinders also appear in the structure, mainly in the borders where the thickness is smaller. PS-cylinders reached hundreds of micrometers, and from the AFM images we can estimate an average diameter of about $12 \mathrm{~nm}$ for the cylinders, and a mean distance between two neighbouring cylinders also of $12 \mathrm{~nm}$. However, we need to advise that it exists a certain imprecision in the lateral interaction between the tip and the surface in the phase mode, which can artificially enlarge the dimension of the cylinders [21]. Even so, this is in agreement with accurate measurements of Transmission Electron Microscopy technique (TEM) carried out in similar SEBS films that were deposited on liquid phosphoric acid substrate [7].

In other parts of the sample it is possible to find mixed regions of stripes and droplets, in which it can be observed that the droplets are somehow germinated inside the stripes and then expelled from it (Fig. 3). This spontaneous segmentation of the stripes, probably related to the Rayleigh instability phenomenon, is still far of being completely elucidated. It is not possible to follow the dynamics of the stripe fragmentation because the resultant stripes and droplets are invisible to optical microscopy. However, when the procedure described in Section 2.2 was repeated using smooth silicon as substrate, similar and visible structures, but not well organized,
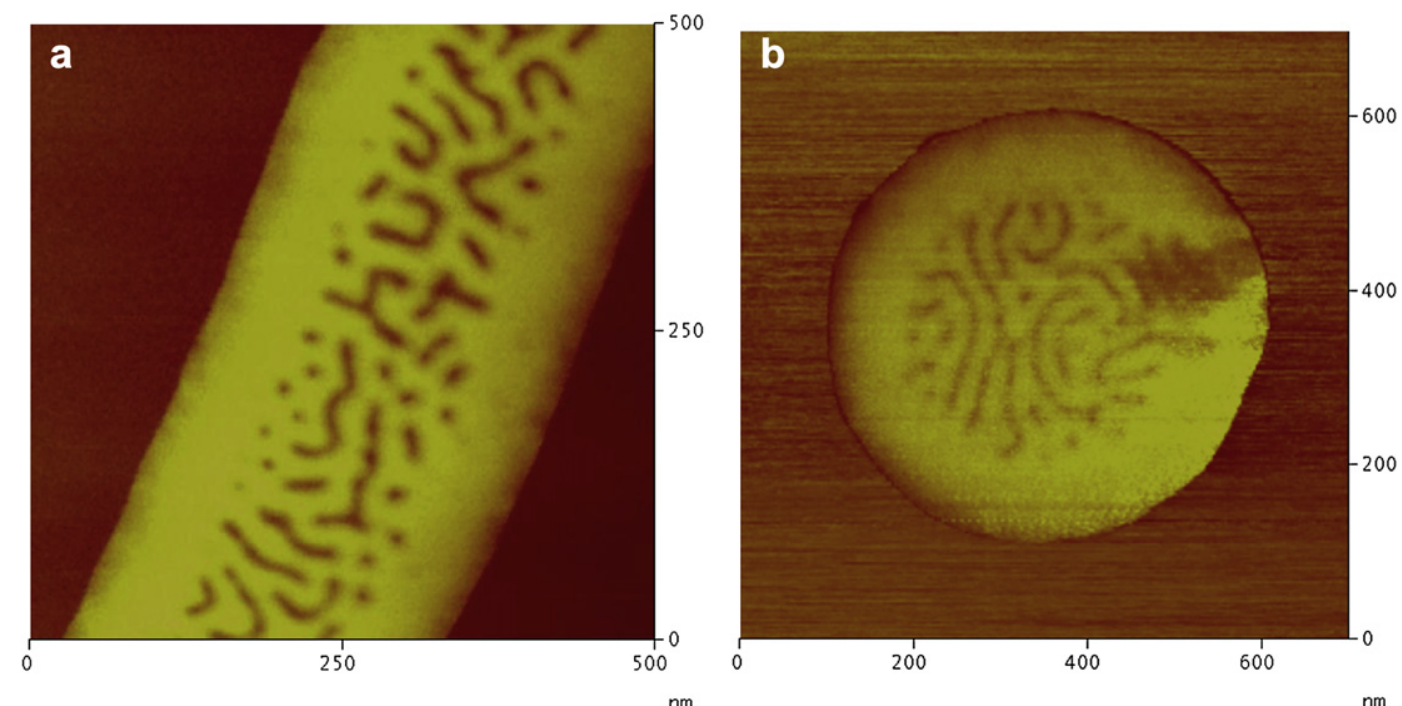

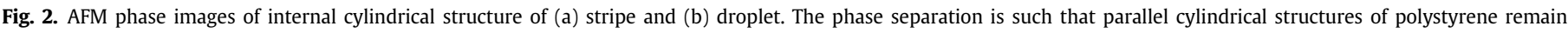
embedded in the poly(ethene-co-butene) matrix. 


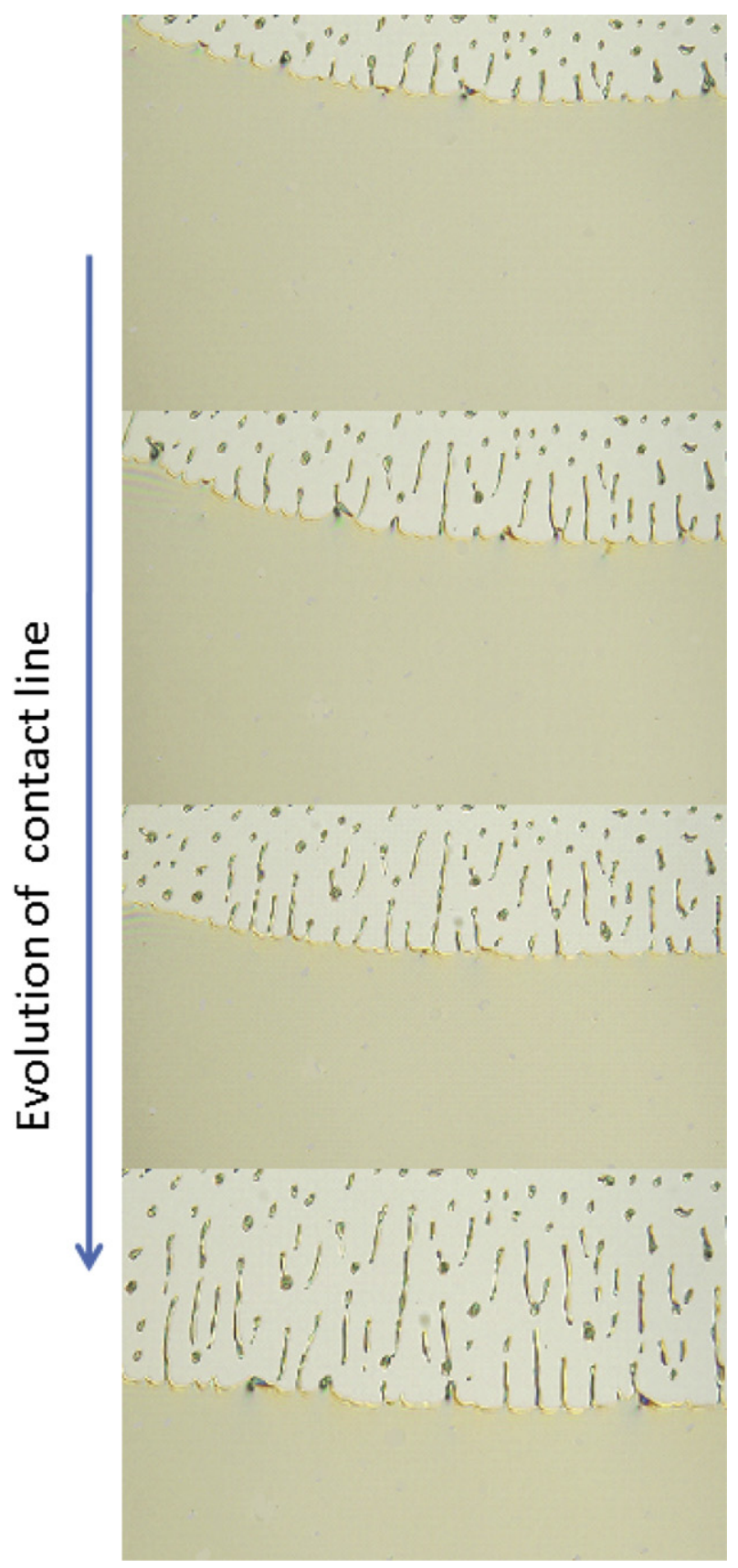

Fig. 3. Optical image of the receding contact line of SEBS toluene thin liquid films onto silicon substrate. The interval between two subsequent pictures was $0.25 \mathrm{~s}$.

were formed. Fig. 3 shows a sequence of optical images during the dewetting process of the SEBS: toluene film. The evolution of the system, as the contact line recedes, shows clearly the formation of fingers and their fragmentation into droplets. This experiment strengthens the hypothesis that the formation of stripes is an intermediate stage for a final droplets pattern.

\subsection{Image analysis}

In possession of the computational tools described above we were able to obtain quantitative data for patterns of droplets and stripes shown in Fig. 1, and also to contribute to the investigation of the stripes segmentation phenomenon (shown in Fig. 4). Fig. 5a

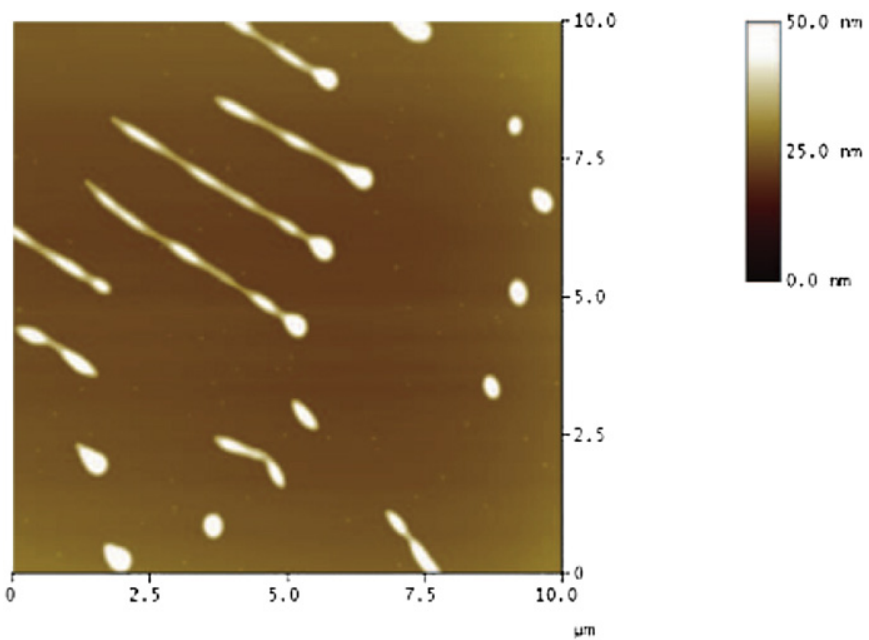

Fig. 4. AFM height image illustrating the stripes fragmentation phenomenon (scales $10 \mu \mathrm{m} \times 10 \mu \mathrm{m} \times 50 \mathrm{~nm}$ ) of SEBS on a mica substrate.

shows a surface height picture extracted from Fig. 1c using the Matlab version 7.8, while in Figs. 5b-d we present, respectively, graphs of height and width for the droplets. The colour gradient facilitates the visual analysis of the pattern. These results clearly show the regularity of the droplets pattern and the uniformity of their shape. Inserts in Fig. 5b and c present the data displayed in the histograms, where we can verify the relatively narrow dispersions for these parameters. The average values for height, diameter and area were found to be $39 \pm 1 \mathrm{~nm}, 520 \pm 20 \mathrm{~nm}$ and $0.20 \pm 0.09 \mu \mathrm{m}^{2}$, respectively. Fig. 6 shows the stripe pattern by the same Matlab treatment (Fig. 1a), in which we can see a higher dispersion in the height distribution, therefore differently from the droplet pattern. The average value for the height of stripes is $23 \pm 2 \mathrm{~nm}$. The geometric measurements of typical structures formed on a mica substrate are compared in Table 1 . However, this height value is slightly different from stripe to stripe because, as we discuss below, the droplets germination starts still inside the stripes. It is important to address here two important points. First, the existence of a height gradient is clearly visible from one extremity of the picture to the other, as shown in Fig. 6. This indicates that for any reason there is transport of mass along each stripe. Second, the height average for stripes is lower than that for droplets.

In order to gain more information about the morphometric parameters of these structures we applied the calculation given above to a transition region, i. e. a region that exhibits stripes and droplets. The pattern shown in Fig. 7a $(60 \times 60 \mu \mathrm{m} 2)$ displays an example of such region. This analysis was carried out in the expanded region shown in Fig. 7b and $\mathrm{c}$ a typical height profile of one stripe shows that it presents a height gradient. This gradient is more visible in the image built up by the computational manipulation of the structure shown in Fig. 7b (shown in Fig. 8), in agreement with what was already observed in Fig. 6. This gradient is more visible in the image built up by the computational manipulation of the structure shown in Fig. 7b (shown in Fig. 8), in agreement with what was already observed in Fig. 6 . This gradient somehow continues along the droplets that were formed from the stripes segmentation, as shown in the graph of Fig. 8. These results reinforce the hypothesis that the morphology of droplets starts to be germinated inside the stripes, and that the final morphology of droplets ends after the segmentation process. In contrast with the values exhibited in Table 1, the height average values for stripes and droplets in the transition region are, respectively, $30 \mathrm{~nm}$ and $35 \mathrm{~nm}$. 

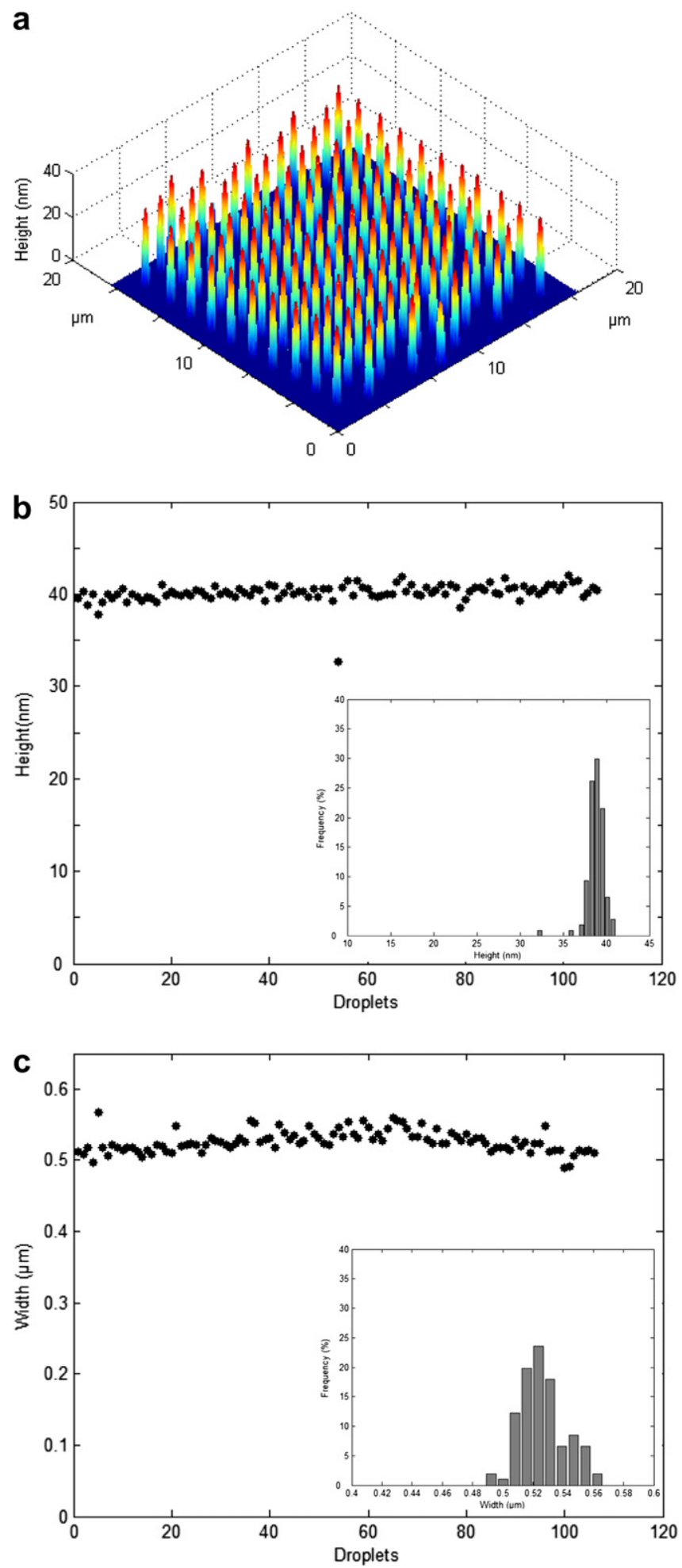

Fig. 5. (a) 3D surface image of droplets of Fig. 1a, where colour gradient represents height values. (b) Height and (c) width of droplets patterns, respectively. Insertions into Fig. $5 \mathrm{~b}$ and $\mathrm{c}$ represent the data displayed in histograms.

\section{Discussion}

As described in the introduction, the formation of fingers during the dewetting of SEBS/toluene thin films onto mica substrates is caused by a Marangoni effect at the contact line.

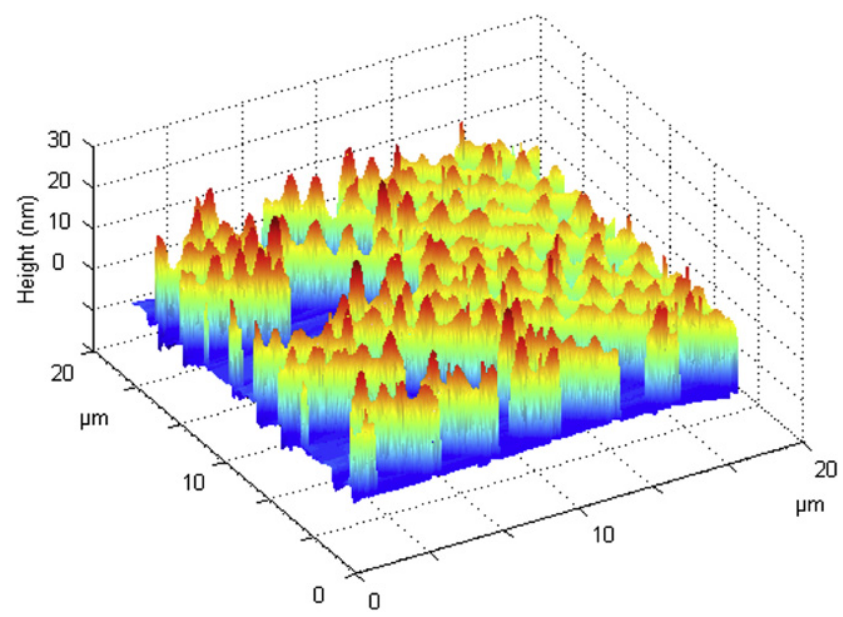

Fig. 6. 3D surface stripes image of Fig. 1c, the colour gradient represents height values.

There upon a periodic instability occurs along each finger (stripe), as can be seen in Figs. 3 and 4. This effect is similar to the Rayleigh-Plateau phenomenon [22]. In a good approximation we can consider a flat rectangular geometry for the stripes with a cross area defined by a width $l \approx 400 \mathrm{~nm}$ and a thickness $d_{s} \approx 23 \mathrm{~nm}$. The established wavy perturbation of wavelength $\lambda$ causes a mass transport from the troughs to the crests, which induced the fragmentation of the stripes into droplets (nanometric discs). Being $\pi b^{2}$ the area of each droplet, where $b$ is the radius, and $d_{d}$ its thickness, by the volume conservation condition, $\lambda$ is equal to $\left(\pi b^{2} / l\right)\left(d_{d} / d_{s}\right)$. The free energy per wavelength for stripes can be considered as $\lambda l\left(\gamma_{s}+\gamma_{g}\right)$, and for the each droplet $\pi b^{2}\left(\gamma_{s}+\gamma_{g}\right)$, where $\gamma_{s}$ is the surface tension at the interface with the substrate, and $\gamma_{g}$ that in contact with the air. Since the condition for the fragmentation is the reduction of the free energy, $\lambda$ should be higher than $\left(\pi b^{2} / l\right)$, or $d_{d}>d_{s}$. This condition is in agreement with the data shown in Table 1.

The question now is: how are the modifications in the internal morphology when stripe segments evolve into droplets? First, we should consider that the internal morphology of such SEBS structures (stripes and droplets) is formed by layers (terraces) in which spaced glassy cylinders of polystyrene, disposed in parallel to the substrate, are immersed in a rubbery matrix of poly(ethene-cobutene) [12,23], with an autophobic PS-layer beneath. This model is proposed by analogy with models for SEBS thin films and similar copolymers $[12,21,23,24]$. It has been extensively discussed in the literature that depending on the surface field PS-cylinders lie aligned perpendicular or parallel to the substrate surface. The images of Fig. 2 indicate that for stripes and droplets the cylinders lie rather with their axis parallel to the substrate, despite the existence of some perpendicular cylinders. Therefore, we assume that the height of each stripe (droplet) can be defined by stacked terraces added to the bottom autophobic layer, as depicted in Fig. 9. For a stripe that has an average height equal to $23 \mathrm{~nm}$ the height is $d_{s}=h+d_{t}$, where $d_{t}$ is the top-terrace thickness, while each droplet

Table 1

Geometrical parameters measurement of self-assembled copolymer, stripes and droplets: the mean height, width and area for objects.

\begin{tabular}{llll}
\hline Objects & $\begin{array}{l}\text { Mean height } \\
(\mathrm{nm})\end{array}$ & $\begin{array}{l}\text { Width } \\
(\mu \mathrm{m})\end{array}$ & $\begin{array}{l}\text { Area } \\
\left(\mu \mathrm{m}^{2}\right)\end{array}$ \\
\hline Stripes (Fig. 1a) & $23 \pm 2$ & $0.406 \pm 0.005$ & - \\
Droplets (Fig. 1b) & $39 \pm 1$ & $0.53 \pm 0.02$ & $0.22 \pm 0.02$ \\
\hline
\end{tabular}



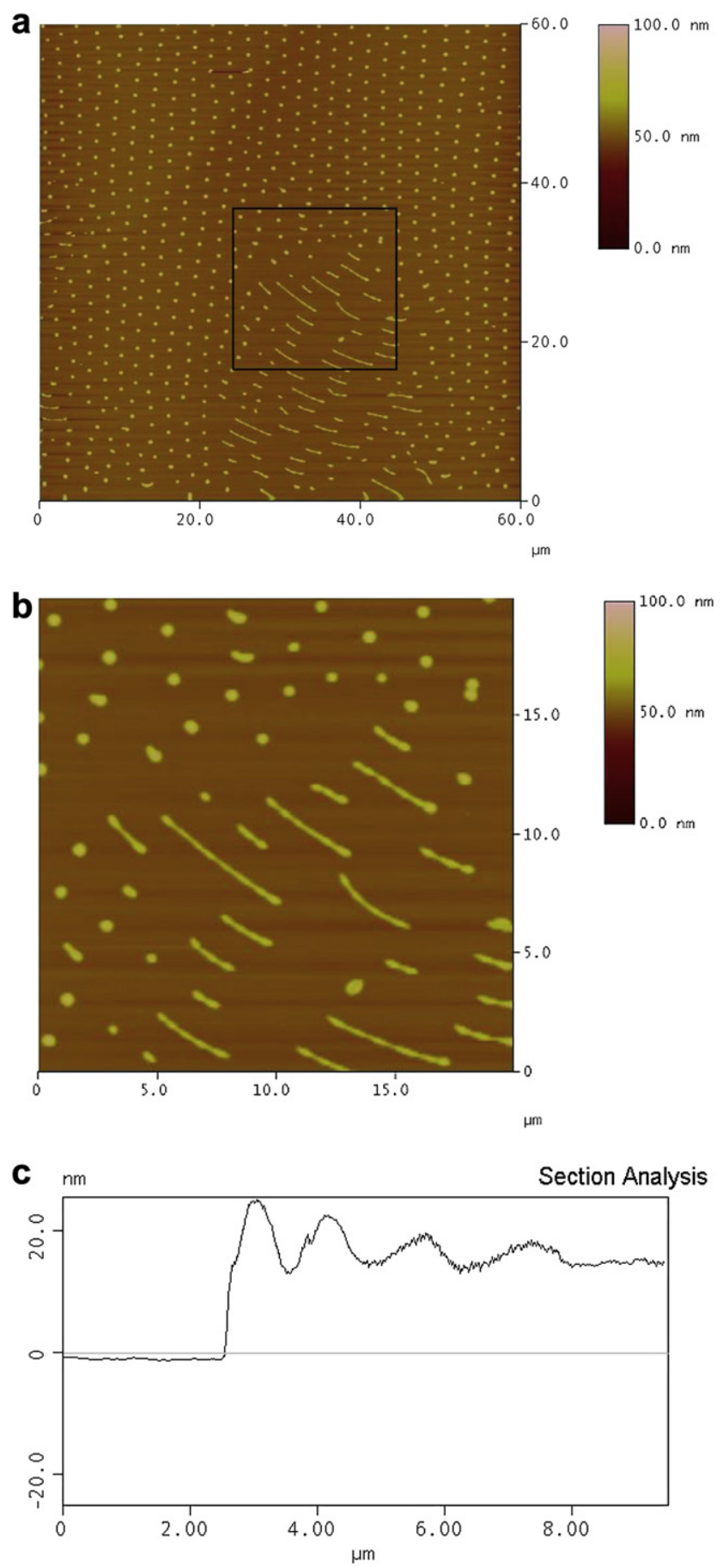

Fig. 7. (a) AFM height image of SEBS copolymer illustrating a transition region, i.e. region that exhibits stripes and droplets, (b) zoomed image obtained from dark region of Fig. 7a, and (c) the illustration of a stripe section analysis in a transition region.

has in addition an intermediate terrace with thickness $d_{i}$, having a total height given by $d_{d}=h+d_{i}+d_{t}$, whose experimental value is $39 \mathrm{~nm}$.

Therefore, the thickness of the internal lamella $d_{i}$ is $16 \mathrm{~nm}$, which is lower than the distance between two cylinders at the surface $(24 \mathrm{~nm})$, but we need to make the projection since the cylinders packing form a triangular arrangement. To sustain this model we need to assume that along the $z$-direction the internal structure is under a compression caused by interfacial interactions.

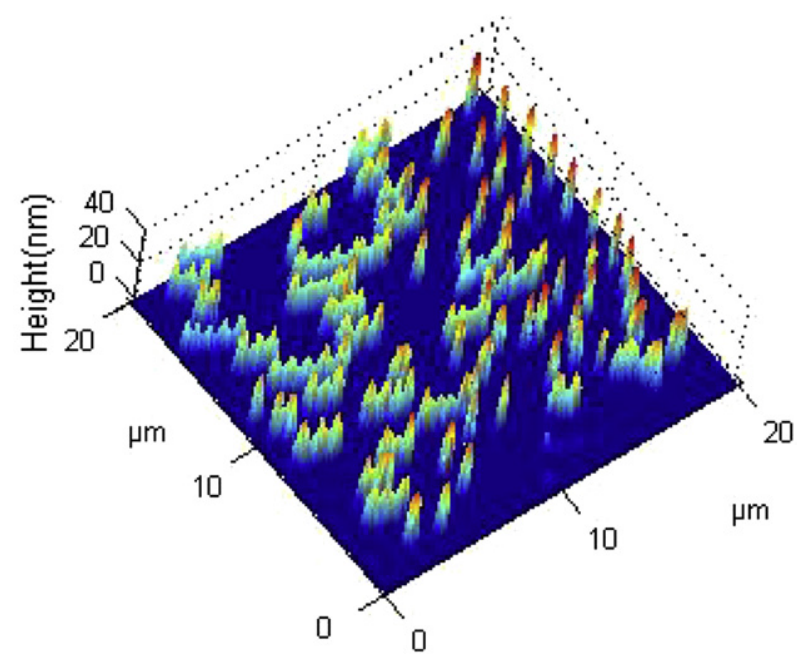

Fig. 8. 3D surface of transition region presented in Fig. $7 \mathrm{~b}$, the colour gradient represents height values.

Being $d_{t}$ approximately equal to $d_{i}, h$ results to be equal to $7 \mathrm{~nm}$. However, based on the model of Fig. 9, $d_{t}$ is slightly lower than $d_{i}$, and $\mathrm{h} \leq 7 \mathrm{~nm}$.

\section{Conclusions and final remarks}

A summary explanation of the structures formation shown in Figs. 1 and 2 can be given in three steps. First, the Marangoni effect occurs in the vicinity of the contact line during the dewetting of the thin film liquid (still a film in solution), an effect that can be generated by a local gradient of the solute (SEBS molecules) with the contribution of the solvent evaporation [10]. In a second step, the undulations along the contact line triggers the formation of fingers, and as the film shrinks these fingers are stuck in a parallel arrangement on the mica surface composing the pattern of stripes (Fig. 1a); this was corroborated by the optical images presented in Fig. 3. The third step, which is the main contribution of this paper, is related to a particular sort of Rayleigh instability, in which the stripes break up in droplets [25]. It is also known that in thin films of SEBS and similar copolymers the phase separation forms a cylindrical domain structure, and for thin films the cylinders can be oriented in parallel to the solid surface [5,12]. Other studies showed that in very thin films the orientation of the cylinders is perpendicular, while for thicker films (above $100 \mathrm{~nm}$ ) they are disposed in parallel to the substrate $[12,23]$. We proposed a model for the morphologies of the stripe and droplet structures, in which
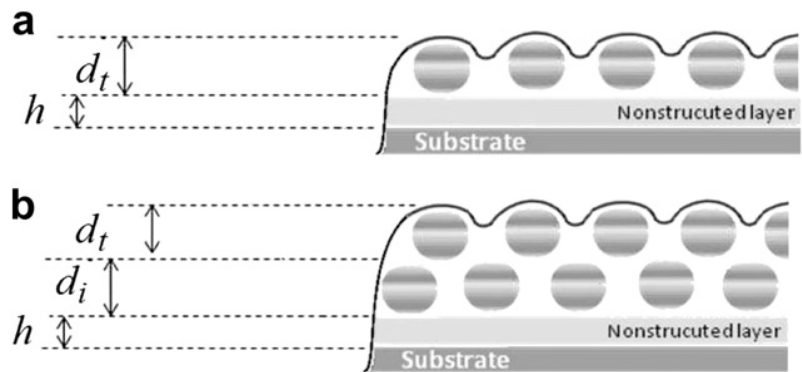

Fig. 9. Illustration of formed SEBS thin film over mica substrate, $h$ is the non structure layer, result of mica copolymer interaction. (a) Represents a transversal profile of a stripe in which lamella $d_{t}$ is the top-terrace thickness, and (b) the profile of a droplet formed by an additional terrace of thickness $d_{i}$. 
the only difference is in the number of terraces formed by PScylinders. This model was obtained from AFM of SEBS images that show parallel arrangement of PS-cylinders. Computational image processing was essential to elaborate the model because provided accurate quantitative values for the heights of stripes and droplets, as well as the height variation in transition regions that showed the stripe fragmentation into droplets.

\section{Acknowledgment}

This work was sponsored by CNPq, Fapemig, FAPESP (Proc. No. 02/07368-9), Capes, and the National Institute of Science and Technology of Organic Electronics (INEO).

\section{References}

[1] Hamley IW. Nanotechnology 2003;14:R39-45.

[2] Segalman RA. Mater Sci Eng R 2005;48:191-226.

[3] Lee G, Jo PS, Yoon B, Kim TH, Acharya H, Ito H, et al. Macromolecules 2008;41:9290-4.

[4] Xu C, Fu XF, Fryd M, Xu S, Wayland BB, Winey KI, et al. Nano Lett 2006;6: $282-7$.
[5] Karthaus O, Koito T, Shimomura M. Mater Sci Eng C 1999;8-9:523-6.

6] Liu Y, Li M, Bansil R, Steinhart M. Macromolecules 2007;40:9482-90.

[7] Wang Y, Shen JS, Long CF. Polymer 2001;42:8443-6.

[8] Sierra CA, Galán C, Fatou JG, Parellada MD, Barrio JA. Polymer 1997;38 (17):4325-35.

[9] Carvalho AJF, Pereira-da-Silva MA, Faria RM. Eur Phys J E 2006;20: 309-15.

[10] Karthaus O, Grasjö L, Maruyama N, Shimomura M. Chaos 1999;9:308-14.

[11] Han X, Hu J, Liu H, Hu Y. Langmuir 2006;22:3428-33.

[12] van Dijk MA, van der Berg R. Macromolecules 1995;28:6773-8.

[13] Kim G, Libera M. Macromolecules 1998;31:2569-77.

[14] Rehse S, Mecke K, Magerle R. Phys Rev E 2008;77:51805-13.

[15] Martín-Palma RJ, Pascual L, Herrero P, Martinez-Duart JM. Appl Phys Lett 2002;81:25-7.

[16] Mahadevan S, Casadent D. Ultramicroscopy 2003;96:153-62.

[17] Oh S, Hyon C, Sull S, Hwangb S, Park Y. Rev Sci Instrum 2003;74:4805-11.

[18] Israelachvili JN, Alcantar NA, Maeda N, Mates TE, Ruths M. Langmuir 2004;20: $3616-22$.

[19] Gonzalez RC. In: Woods RE, editor. Digital image processing. 2nd ed. AddisonWesley; 1992.

[20] Motomatsu M. Polymer 1997;38(8):1779-85.

[21] Wang Y, Song R, Li Y, Shen J. Surf Sci 2003;530:136-48.

[22] Rayleigh L. Proc London Math Soc 1878;10:4-12.

[23] Knoll A, Magerle R, Krausch G. J Chem Phys 2004;120:1105-15.

[24] Epps TH, DeLongchamp DM, Fasolka MJ, Fischer DA, Jablonski EL. Langmuir 2007;23:3355-62.

[25] Reiter G. Langmuir 1993;9:1344-51. 